CLINICAL STUDY

\title{
Activating transcription factor 3: a hormone responsive gene in the etiology of hypospadias
}

\author{
Ana Beleza-Meireles ${ }^{1}$, Virpi Töhönen ${ }^{1}$, Cilla Söderhäll ${ }^{1}$, Christian Schwentner ${ }^{2}$, Christian Radmayr ${ }^{2}$, \\ Ingrid Kockum $^{3}$ and Agneta Nordenskjöld ${ }^{1,4}$ \\ ${ }^{1}$ Department of Molecular Medicine and Surgery, Karolinska Institutet, SE-171 76 Stockholm, Sweden, ${ }^{2}$ Department of Paediatric Urology, University of \\ Innsbruck, 35A-6020 Innsbruck, Austria, ${ }^{3}$ Department of Clinical Neurosciences, Karolinska Institutet, SE-171 76 Stockholm, Sweden and ${ }^{4}$ Department \\ of Women and Child Health, Astrid Lindgren Children Hospital, Karolinska University Hospital, SE-171 76 Stockholm, Sweden \\ (Correspondence should be addressed to A Nordenskjöld who is now at Department of Molecular Medicine, Building CMM O2, Karolinska University \\ Hospital, Solna, SE-171 76 Stockholm, Sweden; Email: agneta.nordenskjold@cmm.ki.se)
}

\begin{abstract}
Introduction: Hypospadias is a common inborn error of the genital development, whose complex etiology remains elusive. Defects of the androgen metabolism and activity have been found in a subset of boys with hypospadias. Moreover, the balance between androgens and estrogens seems to be important to the proper male genital development. Activating transcription factor 3 (ATF3), an estrogen responsive gene, has been reported to be expressed during sexual development and up-regulated in hypospadic genital skin. We investigated ATF3 as a candidate gene for hypospadias. Material and methods: Genotyping of eight-tagged single nucleotide polymorphisms (SNP)s was performed in 330 boys with hypospadias and in 380 healthy controls. Screening for mutations in ATF3 was conducted in a subset of boys with hypospadias. ATF3 expression was evaluated in the foreskin of boys with hypospadias and in healthy controls and in the human fetal genitalia by immunohistochemistry.

Results: Three common SNPs, spanning a region of about $16 \mathrm{~kb}$ in intron 1 of ATF3, are associated with hypospadias. These SNPs are not linked and their effects are independent. The combination of the three risk SNPs yields the highest significance. Mutation screening identified the gene variant c536A $>\mathrm{G}$ in one patient and $\mathrm{c} 817 \mathrm{C}>\mathrm{T}$ in the $3^{\prime}$-UTR in two other patients. ATF3 expression was evidenced in the developing male urethra.

Conclusions: ATF3 gene variants influence the risk of hypospadias. Its hormonal responsiveness may underlie this risk effect. But also other ATF3-dependent biological aspects, such as cell survival and death, response to stress stimuli, or the control of epithelial-mesenchymal interactions, may be of importance.
\end{abstract}

European Journal of Endocrinology 158 729-739

\section{Introduction}

The development of the male external genitalia is a complex process, comprising genetic programming, cell differentiation, hormonal signaling, enzyme activity, and tissue remodeling, which follows an orderly sequence, occurring in a time- and concentrationdependent way (1). Any disturbance in these processes may lead to hypospadias, a midline fusion defect of the male ventral urethra, defining a continuum, from a malformed urethral meatus at the subcoronal margin, or on the glans penis, to an intersex condition. Hypospadias affects $0.3-7$ of 1000 live male births (2). Despite being so common, its etiology is largely elusive. Familial clustering of hypospadias among firstdegree relatives, as well as twin studies and segregation analysis, have supported a strong heritable component in hypospadias. Genes and environmental factors are believed to influence the risk to this disorder of the male sexual development $(3,4)$.
Androgens and the androgen receptor are essential for the development of the male external genital (5). But defects of the androgen metabolism and activity have been found in only a small subset of patients with hypospadias. On the other hand, the importance of estrogens in male reproductive tract development has been increasingly acknowledged. Functional Estrogen Receptors (ESR)s were detected in differentiating male external genitalia localized at the same structures as the androgen receptors, indicating interactions between effects exerted by the two steroid hormones $(7,8)$; and an excess of estrogens in the developing murine urethra results in an inhibition of the cell proliferation in a dose-dependent manner (9). Moreover, hypospadias has been induced in a mice model by maternal exposure to synthetic estrogens during pregnancy (10). And polymorphisms in the gene for the estrogen receptor 2 have been associated with hypospadias in humans (11). It has therefore been proposed that hypospadias and other disorders of the otherwise androgen-dependent male sexual development may 
occur due to the occurrence of androgen-estrogen imbalances, which can be influenced by environmental, hormonal, and genetic factors (12-14).

The up-regulation of estrogen-responsive genes has been documented in hypospadias $(15,16)$. From these genes, the activating transcription factor 3 (ATF3), a member of the ATF/CREB family of transcription factors (17), has been the gene with the greatest up-regulation. This gene has also been shown to be generally up-regulated during sexual differentiation (18). This evidence indicates a potential role of ATF3 in hypospadias and lead us to investigate ATF 3 gene sequence in patients with this inborn error of development. We also evaluated the expression pattern of ATF3 in the human developing genitalia and in the genital skin of prepubertal boys with and without hypospadias.

\section{Methods}

\section{Patients and controls}

For sequence analysis, DNA from 330 boys with nonsyndromic hypospadias was analyzed. As control group, we used DNA from ethnically comparable, anonymous samples, constituting 380 healthy voluntary blood donors at Karolinska University Hospital, Sweden. Genomic DNA was extracted from blood using a standard phenol/chloroform protocol.

For expression analysis, 16 human genital skin samples, from ethnically comparable boys, obtained during surgery in Stockholm, were selected: 10 prepubertal boys with hypospadias (6 severe and 4 mild cases) and 6 healthy boys that underwent circumcision. Human genital fetal tissue was also analyzed. The specimens, five males and five females, from the 9th to 15 th weeks of gestation were obtained following elective abortion and showed no signs of maceration or macroscopic abnormality. The age of the fetuses was estimated from crown-rump length, as defined by Mall (19), using the standard tables of Moore (20) and Patten (21). The anogenital structures were removed en bloc from the fresh fetuses and fixed in formalin. The specimens were routinely embedded in paraffin, processed into $4 \mu \mathrm{m}$ serial transverse sections, and dried at $60^{\circ} \mathrm{C}$ overnight.

An ethics committee has approved the protocols for the research project. And it conforms to the provisions of the Declaration of Helsinki in 1995 (as revised in Edinburgh 2000). Subjects, and/or their parents, gave informed consent and patient anonymity was preserved. Gene nomenclature is described according to recommendations by den Dunnen \& Antonarakis $(22,23)$.

\section{SNP selection and typing}

Eight haplotype-tagging SNPs in the ATF3 gene were selected from the genotypes of the CEPH (Centre d'Etude du Polymorphisme Humain) from Utah (CEU) HapMap samples on the HapMap database (http://www.hapmap. org/) using Haploview version 4.0 (Broad Institute of MIT and Harvard, MA, US) (25) with a logarithm of odds (LOD) cut-off of 3.0 and an $r^{2}>0.8$, including only SNPs with a minor allele frequency of $2 \%$ (Table 1 ). SNP typing was performed using a $5^{\prime}$-nuclease TaqMan assay together with fluorescently labeled probes using standard protocols (Applied BioSystems, Warrington, UK). The samples were analyzed on an ABI 7900HT.

\section{Genetic statistics}

To identify potentially associated polymorphisms, we performed general tests of allele $(2 \times 2$ contingency table and a Pearson test with 1-df) and genotype $(2 \times 3$ contingency table with a Pearson test with 2-df) frequency differences among the cases and the controls. Odd ratios (ORs) and OR 95\% confidence intervals were estimated by the Woolf method (24). These analyses were conducted using Statistica 7.0. Further, single-locus and multimarker haplotype association tests were performed using two different algorithms, Haploview version 3.32 (25) and Unphased version 3.0.10 (26). The calculations were performed allowing for missing data estimation. The threshold for rare haplotype frequency identification was 0.005 . The $P$ values were corrected for multiple testing by performing permutations as follows: the trait values were randomly shuffled between subjects 1000 times. In each permutation, the minimum $P$ value is compared with the minimum $P$ value observed in all the analyses in the original dataset. Pair-wise linkage disequilibrium (LD) between polymorphisms was measured by estimating $D^{\prime}$ (the normalized disequilibrium coefficient that ranges from 0 to 1 ); and the squared correlation coefficient $r^{2}$. Hardy-Weinberg equilibrium of the genotype frequencies at each SNP in controls and cases was accessed with Haploview version 3.32 (25).

To determine whether the associated risk gene variants were independently associated, logistic regression analysis was performed using the SAS computer program (SAS Institute Inc., Cary, NC, USA) where the proc logistic command was used. This resulted in a standard logistic regression model in which the regression coefficients were logarithms of the ORs. The models were compared using the log-likelihood ratio test that approximates to a $\chi^{2}$

Table 1 Activating transcription factor 3 (ATF3) markers and their location.

\begin{tabular}{llll}
\hline No. & Marker & \multicolumn{2}{c}{ NCBI position } \\
\hline 1 & rs2137424 & 6264896 & Intron 1 \\
2 & rs3125289 & 6265926 & Intron 1 \\
3 & rs1877474 & 6275670 & Intron 1 \\
4 & rs11119982 & 6282623 & Intron 1 \\
5 & rs10735510 & 6303239 & Intron 1 \\
6 & rs9429889 & 6307308 & Intron 3 \\
7 & rs12070345 & 6308114 & $3^{\prime}$-UTR \\
8 & rs10475 & 6311392 & $3^{\prime}$-UTR \\
\hline
\end{tabular}


distribution with 1-df. Initially, the different genetic models for each marker against each other were tested. The analysis presented in Table 6 only includes data for the best model. In order to get OR estimates for the different genotypes, variables were recoded into design variables.

\section{PCR and sequencing}

DNA from 102 patients was selected for sequencing. This group consisted of 93 boys with non-syndromic hypospadias, 69 Swedish and 24 non-Swedish patients, mostly of Middle Eastern origin: 47 presented a moderate-to-severe phenotype, while the remaining patients have a mild form of hypospadias, and 33 were familial and 60 were sporadic cases. The remaining nine patients presented hypospadias and anal atresia at birth and are of Swedish ethnicity. As control group for this screening, we used 96 of the aforementioned controls. Genomic sequences were obtained from the National Centre for Biotechnology Information (NCBI) and confirmed at Ensembl Genome Browser. Primers flanking the exon/intron junctions were designed by the Primer 3 (http://primer3.sourceforge.net/) program and used to amplify the four exons and exonic-intronic borders, 5'- and 3'-UTR regions, and the putative promoter region of the ATF 3 gene (Table 2). PCRs were performed with DyNAzyme EXT DNA Polymerase (Finnzyme, Espoo, Finland) following the manufacturer protocols. After ExoSap-IT enzyme (USB Europe $\mathrm{GmbH}$, Staufen, Germany) treatment, the PCR fragments were sequenced on both direction using BigDye Terminator v3.1 kit (Applied BioSystems) and analyzed in ABI Prism 3730 Sequencer. Sequence analysis was performed in the program SeqScape v2.5 (Applied BioSystems).

\section{Homology and structure predictions}

The putative homologs of the human ATF 3 were detected by HomoloGene at http://www.ncbi.nlm.nih.gov/. The alignments were made with ClustalW (European

Table 2 Primers used for PCR and sequencing of activating transcription factor 3 (ATF3).

\begin{tabular}{|c|c|}
\hline Fragment & Primers \\
\hline Promoter & $\begin{array}{l}\text { F: 5'-GTG CGT CCA TGT GGA GTG TCC-3' } \\
\text { R: 5'-ATC TGT CCC TCC GCT AGG TTG-3' }\end{array}$ \\
\hline Exon 1 & $\begin{array}{l}\text { F: } 5^{\prime} \text {-GGC TGG TGT GTG TCT CAG TG-3' } \\
\text { R: } 5^{\prime} \text {-TTT AAG CCA GAG TTA TTT CCA AGC-3' }\end{array}$ \\
\hline Exon 2 & $\begin{array}{l}\text { F: } 5^{\prime} \text {-GCT TAT GGG ACT TTT CTC TGA GG-3' } \\
\text { R: } 5^{\prime} \text {-GTT TGT TTC ACT CTC AAC AAC AGC-3' }\end{array}$ \\
\hline Exon 3 & $\begin{array}{l}\text { F: 5'-AGA GCT TTA GTA TTT CGG GGT CTT-3' } \\
\text { R: 5'-CAT TTC TGC TAC CAG CTA CAA GG-3' }\end{array}$ \\
\hline Exon 4a & $\begin{array}{l}\text { F: 5'-AGA GGG TCT GCA TTT TCC TAA AC-3' } \\
\text { R: } 5^{\prime} \text {-GGG ATT CTA GAG GTA CAC AGG A-3'3 }\end{array}$ \\
\hline $3^{\prime}$-UTRa & $\begin{array}{l}\text { F: 5'-GCC ATT GGA GAG CTG TCT TC-3' } \\
\text { R: 5'-TCT GGC CAC CTG AAT ATC TTG-3' }\end{array}$ \\
\hline $3^{\prime}-$ UTRb & $\begin{array}{l}\text { F: } 5^{\prime}-\text { TGG TTT TTC TCA GAT CTG GTT TC- } 3^{\prime} \\
\text { R: } 5^{\prime} \text {-TCT CAG CAA CCG ACA GAT TT-3' }\end{array}$ \\
\hline
\end{tabular}

Bioinformatics Institute (EBI), European Molecular Biology Laboratory (EMBL), Cambridge, UK). Secondary structures were predicted with NNPREDICT, University of California (http://alexander.compbio.ucsf.edu/ nomi/ nnpredict.html). Three-dimensional structure was predicted at Protein Homology/analogY Recognition Engine (http://www.sbg.bio.ic.ac.uk/ phyre) from the Structural Bioinformatics Group, Imperial College, London, UK.

\section{Immunohistochemistry}

The rabbit antibody anti-ATF3 (Santa Cruz Biotechnology, California, US) and the secondary antirabbit antibody (Vector Laboratories Ltd, California, US) were obtained commercially. The tissues were fixed for 5-7 $\mathrm{h}$ in 4\% formalin, washed four times for $10 \mathrm{~min}$ in PBS, and placed thereafter in $70 \%$ EtOH before paraffin embedding. After dewaxing and rehydration, the paraffin sections were treated for antigen retrieval by heating at $98^{\circ} \mathrm{C}$ in $0.1 \mathrm{M}$ Tris ( $\mathrm{pH} \mathrm{9.0)} \mathrm{for} 20 \mathrm{~min}$ after which the slides were allowed to cool to room temperature. The sections were then treated with $1 \mathrm{M}$ $\mathrm{H}_{2} \mathrm{O}_{2}$ for $15 \mathrm{~min}$ in a dark-blocked endogenous peroxidase activity. To block non-specific antibody binding, sections were pre-incubated in $10 \mathrm{mg} / \mathrm{ml}$ BSA (Sigma) containing $10 \%$ goat non-immune serum (Vector) for $40 \mathrm{~min}$. Affinity-purified polyclonal anti-ATF3 was applied to the sections at a 1:800 dilution in $10 \mathrm{mg} / \mathrm{ml}$ BSA and allowed to incubate at $4{ }^{\circ} \mathrm{C}$ overnight in a humid chamber. The sections were incubated with the biotinylated secondary goat anti-rabbit antibody (Vector Laboratories Ltd.) diluted in buffer containing 1\% BSA and $10 \%$ goat serum followed by enzyme conjugate application (avidin-biotin complex, Vector Laboratories Ltd.) and chromogen development (antibody-enzyme conjugate, Vector Laboratories Ltd.). All sections were counterstained with hematoxylin (Merck) for $15 \mathrm{~s}$ before mounting in Kaiser's glycerin gelatin (Merck). Images of immunostained tissues were captured using a Zeiss microscope. Background controls were performed similarly using only the secondary antibody.

\section{Results}

\section{Genotyping}

Genotyping of the eight haplotype-tagging SNPs yielded a success rate of more than $95 \%$. All loci were in HardyWeinberg equilibrium. Single-locus analysis of the allele and genotypes frequencies of patients versus controls indicates that three of the studied polymorphisms in ATF 3 are associated with hypospadias: the T allele and the TT genotype in rs3125289; the T allele and the TT genotype in rs1877474; and the $\mathrm{C}$ allele and the $\mathrm{CC}$ genotype in the rs11119982. The estimated $P$ value after correcting for multiple comparisons by performing 1000 permutations remains below 0.05 only for the 
SNP rs11119982 (Tables 3 and 4). In spite of that, we pursued the analysis of the three SNPs.

The study of haplotype effects, allowing for missing data such as uncertain phase and missing genotypes, has identified that the combination of the three risk alleles in the SNPs rs3125289 (T), rs1877474 (T), and rs11119982 (C) actually yields the higher effects when compared with the analysis of the alleles individually or when combined two-by-two; and the combination of TTC is the most strongly associated with hypospadias in our study (Table 5). The estimated $P$ value after correcting for multiple comparisons by performing 1000 permutations remains significant. The $D^{\prime}$, the normalized disequilibrium coefficient, is very low (0.06) evidencing that the alleles are not in LD. Other marker combinations were not significantly associated with hypospadias.

In order to test if the risk effect of the three risk alleles in the SNPs rs3125289 (T), rs1877474 (T), and rs11119982 (C) were independently associated with hypospadias, we used logistic regression analysis. As the $P$ value for the presence of the risk alleles was the lowest, we used co-dominant coding when comparing with the fit of models containing the three risk markers. Three models were tested, one with only one polymorphism, one with two polymorphisms, and one with the three polymorphisms together. All the tested models yielded significant $P$ values. The model containing the three risk alleles performs better (lowest $P$ value) than the other two models (Table 6). However, the test including interaction terms did not perform better than the previous test, suggesting that these three gene variants are not in epistasis, and their conjoined effect may be only additive.

The control population constituted of healthy blood donors in Stockholm, which includes individuals from this ethnicity. However, we cannot access in detail this population, since it is totally anonymous, which could represent a bias to our study. However, we controlled this problem by analyzing separately the different ethnic groups within the patient groups. And the results are independent of the ethnical origin. The allele frequencies are similar in the different groups; $\chi^{2}$ test to access differences in frequencies yielded non-significant $P$ values (data not shown).

\section{Mutation screening}

Mutation screening performed in 93 patients and 96 controls revealed several genetic variants (Fig. 1). A missense mutation in exon $3, \mathrm{c} 536 \mathrm{~A}>\mathrm{G}$, causing an amino acid substitution, Arg90Gly, was found in one Swedish patient with a sporadic moderate form of hypospadias. An 3'-UTR gene variant, c817C $>$ T, was found in two patients, one Swedish patient with a sporadic form of hypospadias and one boy from Middle Eastern with a familiar form, both moderate-to-severe phenotypes. The three affected boys are heterozygous for the risk alleles in rs1877474 and rs11119982. These newly identified gene variants were not found in the control population.

Table 3 Cases $(\mathrm{Ca})$ versus controls $(\mathrm{Co})$ study for single-locus analysis: three of the studied polymorphisms in activating transcription factor 3 (ATF3) are associated with hypospadias: rs3125289, rs1877474, and rs11119982.

\begin{tabular}{|c|c|c|c|c|c|c|c|}
\hline SNP & Genotype & Case & Control & Ca-Freq & Co-Freq & $P$ value & OR $(95 \% \mathrm{Cl})$ \\
\hline \multirow[t]{3}{*}{ rs2137424 } & $\mathrm{CC}$ & 34 & 47 & 0.1164 & 0.1288 & & \\
\hline & $\mathrm{CT}$ & 124 & 152 & 0.4247 & 0.4164 & & \\
\hline & TT & 134 & 166 & 0.4589 & 0.4548 & 0.8901 & NS \\
\hline \multirow{3}{*}{ rs3125289 } & CC & 53 & 101 & 0.2181 & 0.2708 & & \\
\hline & CT & 113 & 189 & 0.465 & 0.5067 & & \\
\hline & TT & 77 & 83 & 0.3169 & 0.2225 & 0.02831 & TT: $1.62(1.13-2.33)$ \\
\hline \multirow[t]{3}{*}{ rs1877474 } & $\mathrm{CC}$ & 46 & 81 & 0.1365 & 0.2166 & & \\
\hline & CT & 157 & 171 & 0.4659 & 0.4572 & & \\
\hline & TT & 134 & 122 & 0.3976 & 0.3262 & 0.01108 & TT: 1.36 (1.01-1.85) \\
\hline \multirow[t]{3}{*}{ rs11119982 } & CC & 82 & 60 & 0.2485 & 0.1604 & & \\
\hline & CT & 160 & 168 & 0.4848 & 0.4492 & & \\
\hline & TT & 88 & 146 & 0.2667 & 0.3904 & $4 \times 10^{-4}$ & CC: $1.73(1.19-2.51)$ \\
\hline \multirow[t]{3}{*}{ rs10735510 } & AA & 91 & 127 & 0.2809 & 0.3396 & & \\
\hline & $A C$ & 158 & 179 & 0.4877 & 0.4786 & & \\
\hline & $\mathrm{CC}$ & 75 & 68 & 0.2315 & 0.1818 & 0.1327 & NS \\
\hline \multirow[t]{3}{*}{ rs9429889 } & $\mathrm{CC}$ & 235 & 285 & 0.7015 & 0.756 & & \\
\hline & $\mathrm{CT}$ & 90 & 86 & 0.2687 & 0.2281 & & \\
\hline & TT & 10 & 6 & 0.02985 & 0.01592 & 0.1793 & NS \\
\hline \multirow[t]{3}{*}{ rs12070345 } & AA & 79 & 75 & 0.2365 & 0.206 & & \\
\hline & $A G$ & 170 & 174 & 0.509 & 0.478 & & \\
\hline & GG & 85 & 115 & 0.2545 & 0.3159 & 0.1847 & NS \\
\hline \multirow[t]{3}{*}{ rs10475 } & $\mathrm{CC}$ & 221 & 252 & 0.6637 & 0.6649 & & \\
\hline & CT & 96 & 116 & 0.2883 & 0.3061 & & \\
\hline & $\mathrm{TT}$ & 16 & 11 & 0.04805 & 0.02902 & 0.3906 & NS \\
\hline
\end{tabular}

Empirical 5\% quantile of the best $P$ value after 1000 permutations: 0.007799 (Unphased version 3.0.10).

Significant results are in bold. 
Table 4 Cases versus controls study for single-locus analysis: four of the studied polymorphisms in activating transcription factor 3 (ATF3) are associated with hypospadias: rs3125289, rs1877474, rs11119982, and rs10735510.

\begin{tabular}{lllllllll}
\hline SNPs & rs2137424 & rs3125289 & rs1877474 & rs11119982 & rs107355 & rs942988 & rs1207034 & rs10475 \\
\hline A $>$ a & $\mathrm{T}>\mathrm{C}$ & $\mathrm{C}>\mathrm{T}$ & $\mathrm{T}>\mathrm{C}$ & $\mathrm{T}>\mathrm{C}$ & $\mathrm{A}>\mathrm{C}$ & $\mathrm{C}>\mathrm{T}$ & $\mathrm{G}>\mathrm{A}$ & $\mathrm{C}>\mathrm{T}$ \\
'a' cases & 192 & 267 & 249 & 324 & 308 & 110 & 328 & 128 \\
\% 'a' & 32.9 & 54.9 & 36.9 & 49.1 & 47.5 & 16.4 & 49.1 & 19.2 \\
'A' cases & 392 & 219 & 425 & 336 & 340 & 560 & 340 & 538 \\
'a' controls & 246 & 355 & 333 & 288 & 315 & 98 & 324 & 138 \\
\% 'a' & 33.7 & 47.6 & 44.5 & 38.5 & 42.1 & 13 & 44.5 & 18.2 \\
'A' controls & 484 & 391 & 415 & 460 & 433 & 656 & 404 & 620 \\
OR & 0.96 & 1.34 & 0.73 & 1.54 & 1.25 & 1.31 & 1.2 & 1.07 \\
cOR Cl 95\% & $0.75-1.24$ & $1.07-1.68$ & $0.59-0.90$ & $1.25-1.90$ & $1.01-1.53$ & $0.98-1.75$ & $0.98-1.47$ & $0.80-1.44$ \\
CHI SQR & 0.1 & 6.4 & 8.4 & 16 & 4.1 & 3.3 & 3 & 0.2 \\
YATES $\chi^{2}$ & 0.1 & 6.1 & 8.1 & 15.6 & 3.9 & 3.1 & 2.8 & 0.2 \\
P YATES & 0.7518 & 0.0135 & 0.0044 & 0.0001 & 0.0483 & 0.0783 & 0.0943 & 0.6547 \\
Perm* P & NS & NS & 0.0340 & 0.0020 & NS & NS & NS & NS \\
value & & & & & & & &
\end{tabular}

The estimated $P$ value after correcting for multiple comparisons by performing 1000 permutations* remains below 0.05 for rs 1877474 and rs 11119982 . Permutation $P$ value calculated with Unphased and Haploview softwares allowing for missing data such as uncertain phase and missing genotypes. The three affected boys where the mutations $536 \mathrm{~A}>\mathrm{G}$ and $\mathrm{c} 817 \mathrm{C}>\mathrm{T}$ were found are heterozygous for the risk alleles in rs 1877474 and $\mathrm{rs} 11119982$.

Homology analysis of the ATF 3 mRNA revealed that the $\mathrm{c} 536 \mathrm{~A}>\mathrm{G}$ gene variant is located in a highly conserved locus (Fig. 2). Furthermore, protein structure prediction suggests that this variant is predicted to induce a change in the protein structure (Fig. 3; detail in Fig. 4). This evidence indicates that the variant c536A $>\mathrm{G}$ is likely to have a functional consequence. Analysis of the $\mathrm{c} 817 \mathrm{C}>\mathrm{T}$ locus has shown that it is not conserved (Fig. 2). However, it is predicted to be a highly regulatory sequence (data not shown). A new polymorphism was found in the promoter region, g.210848306delC, in heterozygous form in 7 out of 93 patients and 8 out of 96 controls. This polymorphism is not associated with hypospadias $(P=0.3)$.

\section{Immunohistochemistry}

ATF3 protein expression in human prepubertal samples ATF3 protein expression is observed at the nuclei of stromal cells and the vascular endothelium in foreskin tissue samples from prepubertal boys with hypospadias and in healthy controls. The number of positive cells or the staining intensity did not differ notably between the patient group and the controls (Fig. 5). In a few samples from patients and controls, some positively stained cells could be observed in the epidermal region. The ATF3 staining was then seen in both nuclei and cytoplasm in these cells of different origins (Fig. 6).

\section{ATF3 protein expression in human fetal samples}

Human fetal sections from gestational ages 9-15 weeks were analyzed for ATF3 expression by immunohistochemistry. ATF 3 expression is seen in the urethral wall at 15th week of gestation particularly in the nucleus of epithelium cells, preferentially adjacent to the nuclear membrane (Fig. 7). A similar pattern of expression was observed in the developing vagina at every observed stage. Nuclear ATF3 expression was observed consistently in the rectum walls in both male and female fetuses.

Table 5 Study of haplotype effects, allowing for missing data such as uncertain phase and missing genotypes.

\begin{tabular}{lccllll}
\hline & \multicolumn{5}{c}{ Estimates of haplotype effects (Reference C-T-T) } \\
\cline { 2 - 7 } Haplotype & Case & Control & Ca-Freq & Co-Freq & $\chi^{2}$ & $P$ value \\
\hline C-C-C & 70.1 & 52.34 & 0.1028 & 0.06906 & 0.4544 & 0.5003 \\
C-C-T & 49.66 & 87.4 & 0.07282 & 0.1153 & $\mathbf{1 5 . 2 1}$ & $\mathbf{9 . 6 4 \times 1 0 ^ { - 2 a }}$ \\
C-T-C & 73.39 & 87.19 & 0.1076 & 0.115 & 3.53 & 0.06025 \\
C-T-T & 113.2 & 170.5 & 0.166 & 0.2249 & 7.957 & 0.004789 \\
T-C-C & 94.9 & 129.6 & 0.1392 & 0.171 & 0.03103 & 0.8602 \\
T-C-T & 37.52 & 68.89 & 0.05501 & 0.09088 & 11.95 & $5.5 \times 10^{-2}$ \\
T-T-C & 96.99 & 22.99 & 0.1422 & 0.03033 & $\mathbf{5 6 . 8 9}$ & $\mathbf{4 . 6} \times \mathbf{1 0}$ \\
T-T-T & 146.2 & 139 & 0.2144 & 0.1834 & 1.828 & 0.1763 \\
\hline
\end{tabular}

The reference haplotype was CTT, the most common haplotype in the control population. The combinations of the three risk alleles in the ATF3, SNPS rs3125289 (T), rs1877474 (T), and rs11119982 (C) yield the higher effects in our study. The empirical $5 \%$ quantile of the best $P$ value is 0.002013 after 1000 permutations. $D^{\prime}=0.06$. Ca, case; Co, control.

${ }^{\text {aP }}$ utative protective haplolype.

bPutative susceptibility haplotype.

Significant results are in bold. 
Table 6 Logistic regression modeling to evaluate the interaction between risk SNPs: rs3125289, rs1877474, and rs11119982.

\begin{tabular}{|c|c|c|c|c|}
\hline & Intercept only & $\begin{array}{l}\text { No interaction } \\
\text { assumption }\end{array}$ & $\begin{array}{l}\text { Assumption of two- } \\
\text { by-two interaction }\end{array}$ & $\begin{array}{c}\text { Assumption of all SNPs } \\
\text { interacting }\end{array}$ \\
\hline \multicolumn{5}{|l|}{ Model fit } \\
\hline $\mathrm{AIC}$ & 811.989 & 782.056 & 775.322 & 770.718 \\
\hline SC & 816.393 & 799.671 & 788.533 & 779.526 \\
\hline$-2 \log L$ & 809.989 & 774.056 & 769.322 & 766.718 \\
\hline \multicolumn{5}{|l|}{ HO test, i.e., $\beta=0$} \\
\hline Likelihood ratio & & 35.9328 & 40.6668 & 43.2707 \\
\hline$P$ & & $7.74 \times 10^{-8}$ & $1.48 \times 10^{-9}$ & $4.77 \times 10^{-11}$ \\
\hline \multicolumn{5}{|l|}{ Factors } \\
\hline \multicolumn{2}{|l|}{ Intercept estimated $\beta$} & -3.1552 & -2.3888 & -1.3969 \\
\hline \multirow{2}{*}{\multicolumn{2}{|c|}{$\begin{array}{l}P \\
\text { Factor } 1\end{array}$}} & $<0.0001$ & $<0.0001$ & $<0.0001$ \\
\hline & & rs3125289 & & \\
\hline \multicolumn{2}{|l|}{ Estimated $\beta$} & 0.2973 & & \\
\hline \multicolumn{2}{|l|}{$P$, Wald stat } & 0.0148 & NS & NS \\
\hline \multicolumn{2}{|l|}{ OR $(05 \% \mathrm{Cl})$} & Not applicable & & \\
\hline \multicolumn{2}{|l|}{ Factor 2} & rs1877474 & & \\
\hline \multicolumn{2}{|l|}{ Estimated $\beta$} & 0.468 & & \\
\hline \multicolumn{2}{|l|}{$P$, Wald stat } & 0.0003 & NS & NS \\
\hline \multicolumn{2}{|l|}{ OR $(05 \% \mathrm{Cl})$} & & & \\
\hline \multicolumn{2}{|l|}{ Factor 3} & rs1111998 & & \\
\hline \multicolumn{2}{|l|}{ Estimated $\beta$} & 0.5852 & 0.5762 & \\
\hline \multicolumn{2}{|l|}{$P$, Wald stat } & $<0.0001$ & $<0.0001$ & NS \\
\hline \multicolumn{2}{|l|}{ OR $(05 \% \mathrm{Cl})$} & & & \\
\hline \multicolumn{2}{|l|}{ Factor 4} & & Two risk alleles & \\
\hline \multicolumn{2}{|l|}{ Estimated $\beta$} & & 0.1998 & \\
\hline \multicolumn{2}{|l|}{$P$, Wald stat } & & $<0.0001$ & NS \\
\hline \multirow{2}{*}{\multicolumn{2}{|c|}{ OR $(05 \% \mathrm{Cl})$}} & & & \\
\hline Factor 5 & & & & Three risk alleles \\
\hline \multicolumn{2}{|l|}{ Estimated $\beta$} & & & 0.1213 \\
\hline \multicolumn{2}{|l|}{$P$, Wald stat } & & & $<0.0001$ \\
\hline \multicolumn{2}{|c|}{ OR $(05 \% \mathrm{Cl})$} & & & Not applicable \\
\hline \multirow{2}{*}{\multicolumn{2}{|c|}{ Ass of predicted and obs responses }} & & & \\
\hline & & 0.129 & 0.135 & 0.124 \\
\hline
\end{tabular}

Significant results are in bold.

\section{Discussion}

Hypospadias is one of the most common inborn errors of development in humans, with complex and still elusive etiology (1-4). Currently hypospadias is repaired surgically, constituting one of the most common surgeries performed in neonates. Although surgery may remain the therapy of choice for hypospadias, a better knowledge of the hormonal and molecular mechanisms of the genital tubercle development may be the basis for preventive strategies to reduce the incidence of this common malformation.
We report three SNPs in the ATF3 sequence, spanning a region of about $16 \mathrm{~kb}$ in intron 1 , which affect the risk for hypospadias. These risk SNPs are not linked and show independent association with hypospadias, as we did not find evidence of epistasis. However, the presence of all three markers yields the most significant result, which indicates an additive effect; it may also suggest that these markers mark a so far unidentified risk increasing gene variant. Additionally, a missense mutation in exon 3 and a sequence variant in the $3^{\prime}$-UTR region of this gene are present in three non-related boys with hypospadias. The first
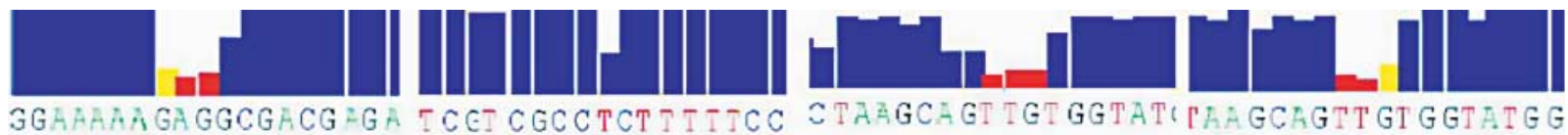

ऽTAAGCAGTTGT GGTATCPAAGCAGTT GT GGTATG G

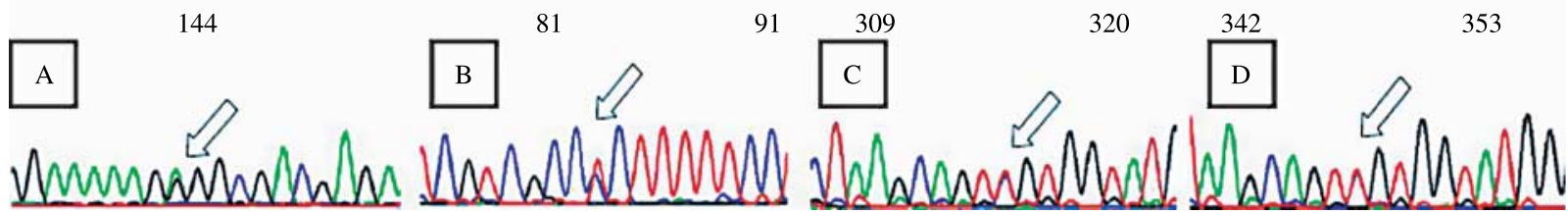

Figure 1 Mutations in ATF3 found in hypospadias patients. 536A $>\mathrm{G}$ in one patient, forward (A) and reverse (B). $3^{\prime}-$ UTR gene variant c817C $>T$ in two other patients $(C$ and $D)$. 


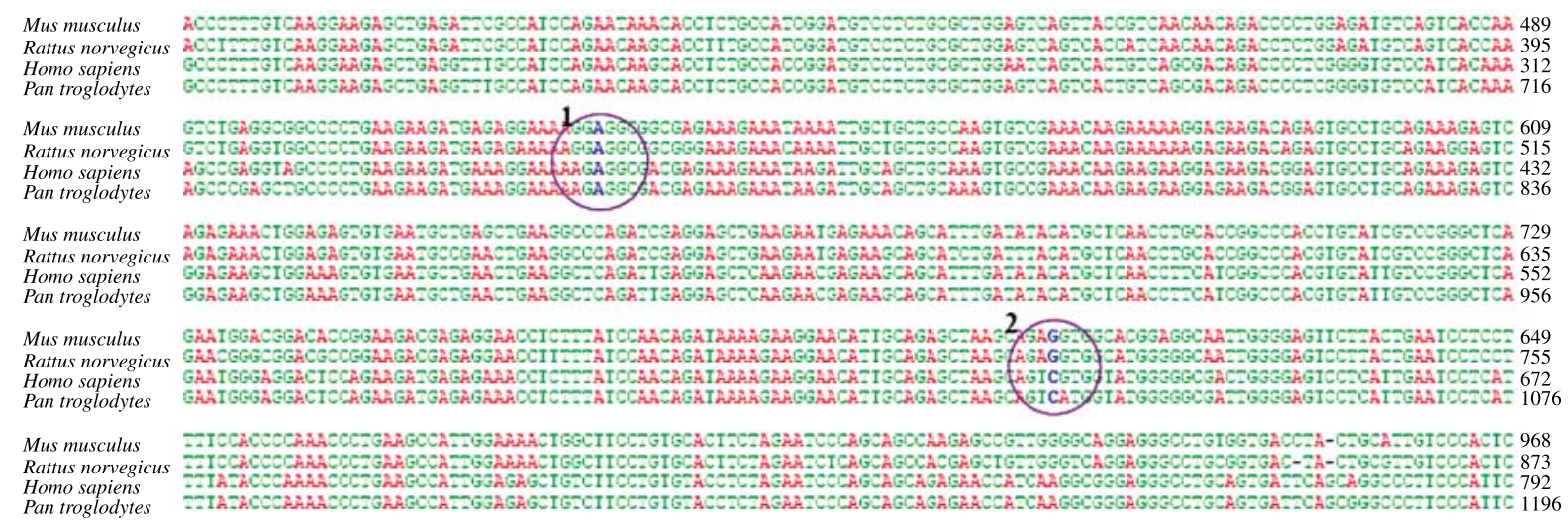

Figure 2 ClustalW homology prediction of ATF3 mRNA. The location of the 536A $>$ G (1) corresponds to a highly conserved region; the location of the UTR mutation c817C > T (2) is only conserved in the Homo sapiens and Pan troglodytes, which is the most phylogenetically closer to the human sequence.

causes an amino acid change that seems to be responsible for a change in the protein structure. The variant located in the $3^{\prime}$-UTR region may affect regulatory events.
We also showed that ATF3 is expressed in the human developing male urethra and in the genital skin of prepubertal boys. This evidence strengthens previous reports implicating ATF3 in genital development and

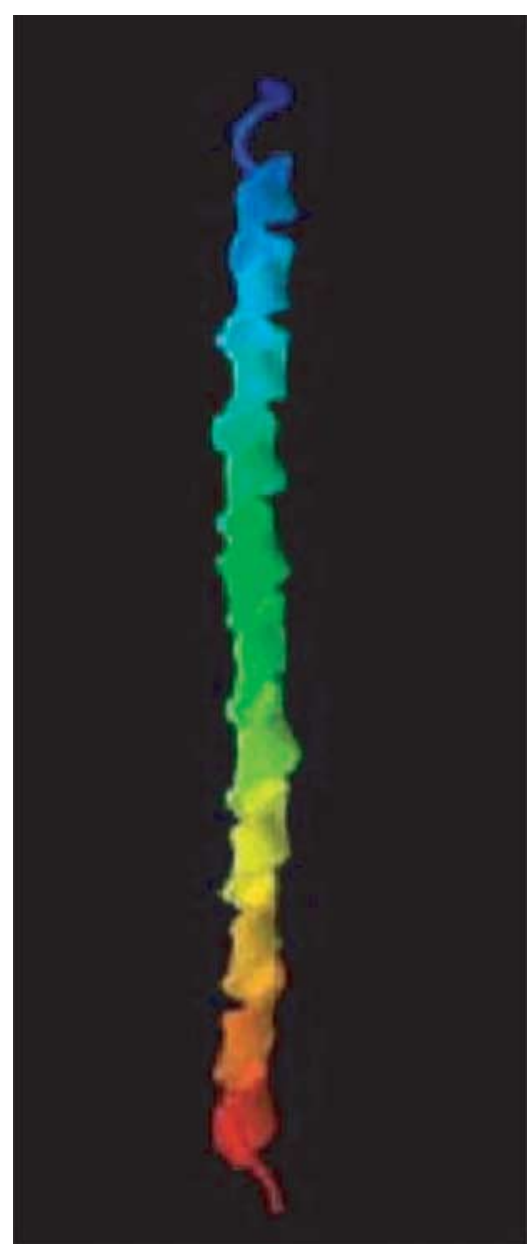

ATF3mut

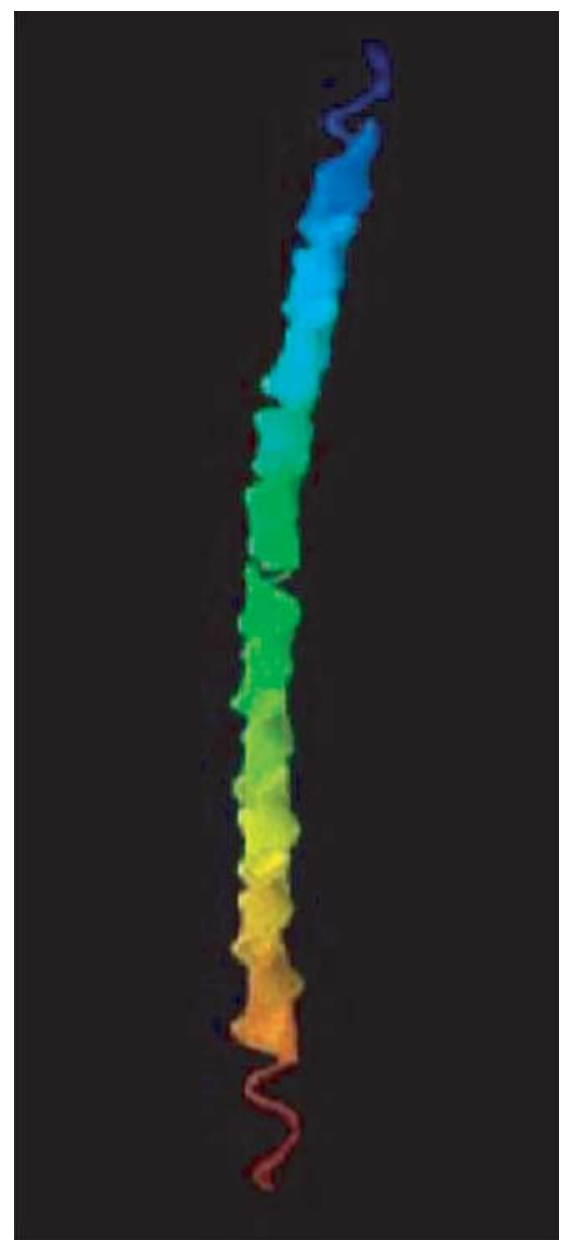

Figure 3 Protein prediction of ATF3 and ATF3 carrying the mutations $536 \mathrm{~A}>\mathrm{G}$ (ATF3 mut). Structure prediction: ATF3 (estimated precision: $95 \%$; $E$ value: 0.0017 ) and ATF3mut (estimated precision: 95\%; Evalue: 0.0039). Predicted at PHYRE-Structural Bioinformatics Group Imperial College, London. A break on the helix in the protein is predicted as consequence of the mutation. 

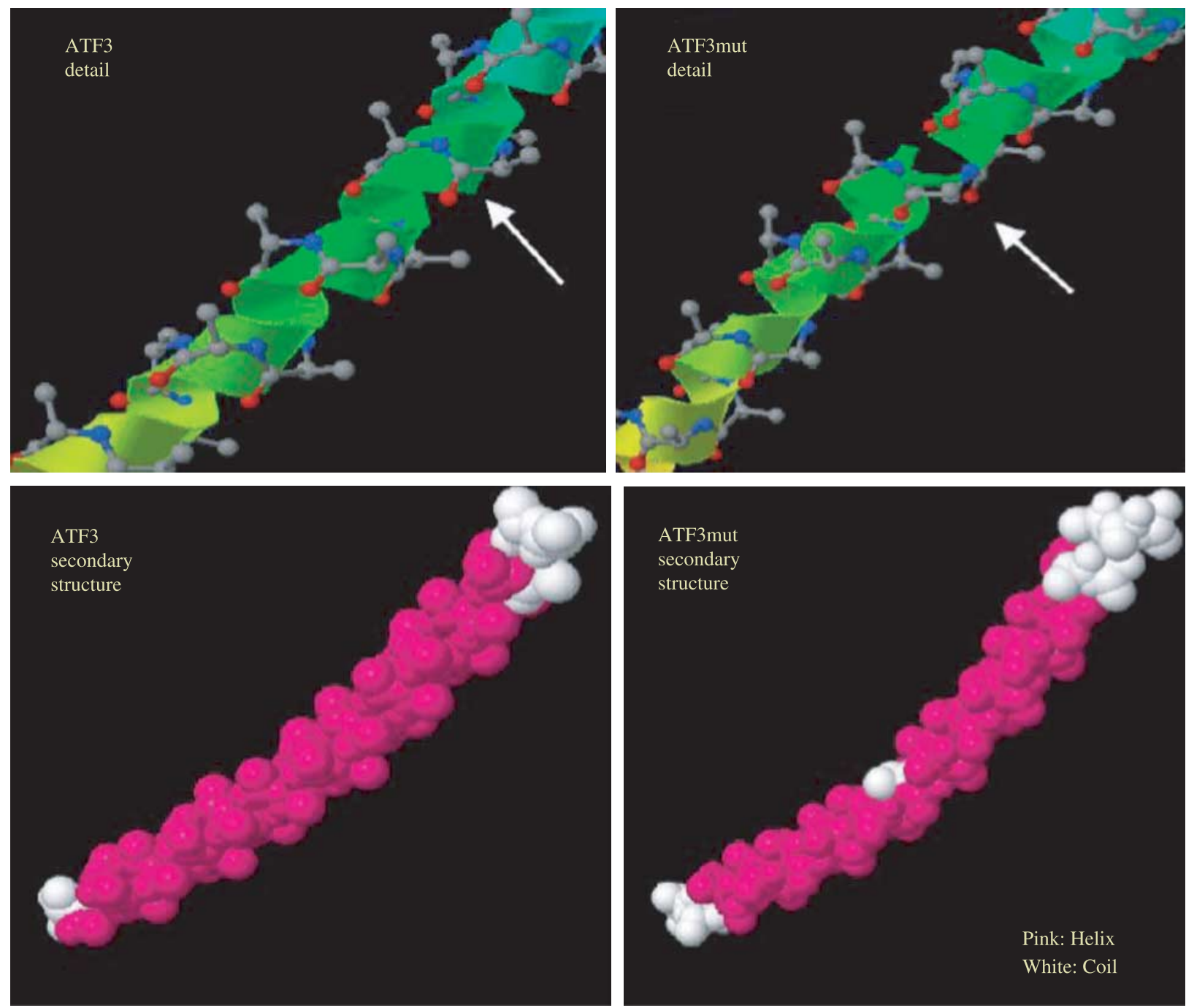

Figure 4 Detail of the protein prediction to ATF3 and ATF3 carrying the mutations 536A $>$ G (ATF3 mut). Predicted at PHYRE-Structural Bioinformatics Group Imperial College, London.
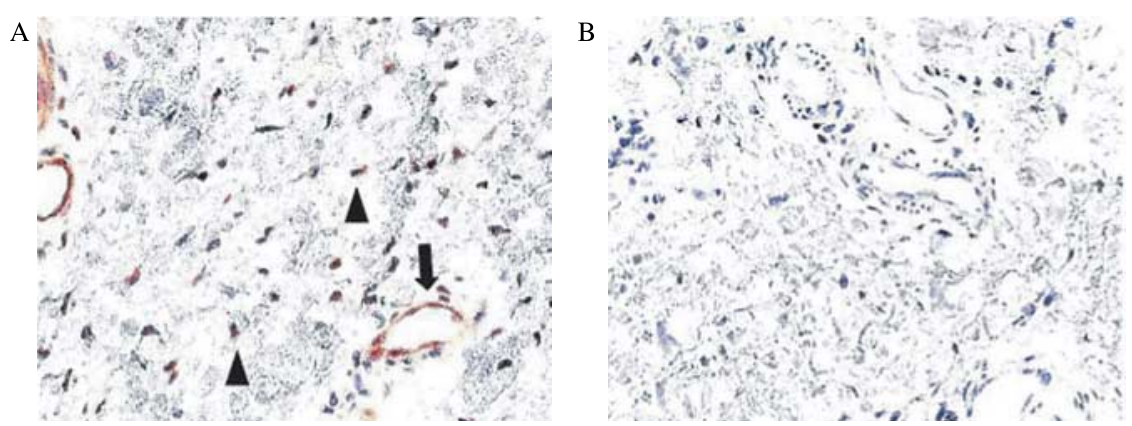

Figure 5 Immunohistochemistry results with ATF3 expression in genital skin tissue. ATF3 expression by immunohistochemistry in human prepubertal genital skin from patients and control individuals. ATF3 expression is localized predominantly in the nuclei in hypospadias patients and healthy controls. Staining in all control and hypospadias individuals was similar, localizing in the nuclei with some predominance around the nuclear membrane in the stromal region of the foreskin (arrows). (A) A representative section of a genital skin tissue from a healthy boy showing positive staining for ATF3 localizing in the nuclei and around the nuclear membrane in the stromal cells (arrowheads) and in the vascular endothelium of the foreskin (arrow). All genital skin tissues from control individuals and hypospadias patients at different ages showed similar staining. (B) The background control using only the secondary antibody (sections photographed at $400 \times$ magnification). 

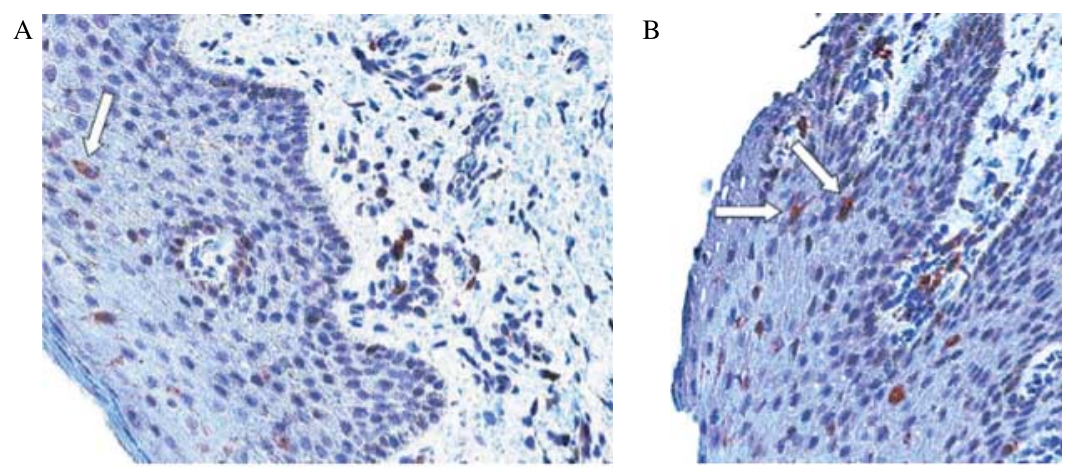

Epidermis

Dermis
Figure 6 ATF3 expression by immunohistochemistry in human prepubertal genital skin from a boy with severe hypospadias. In this specific patient with severe hypospadias ( $A$ and $B$ ), ATF3 expression is observed in both dermal and epidermal cells. The staining is mostly nuclear, but in certain epidermal cells of different origins, ATF3 expression (arrows) is localized in the cytoplasm (sections photographed at $400 \times$ magnification). associates $A T F 3$ sequence variants with an increased risk to hypospadias. The mechanisms that explain how variants in the sequence of ATF3 affect the risk to hypospadias are not yet known; but some hypotheses can be suggested.

Variants in ATF3, a gene that is up-regulated in hypospadias, may affect the risk to this hormonedependent disorder. This effect may be due to disturbances on estrogenic responses during development $(15,16,18)$. Moreover, ATF3 expression has also been reported to be up-regulated by the androgen receptor's activation by both androgens and estrogens in cell lines (27); this aspect indicate that variants in ATF3 may underlie androgenestrogen imbalances and influence the responsiveness to endocrine disrupters. Furthermore, ATF3 is a key immediate early gene induced by gonadotropin-releasing hormone (GnRH) in vivo and in vitro (28). The $\mathrm{GnRH}$ is a key regulator of mammalian reproduction and is required for synthesis and secretion of luteinizing hormone (LH) and follicle-stimulating hormone (FSH), two essential signals in the establishment of primary and secondary sexual characteristics. Interestingly, LH and FSH response to $\mathrm{GnRH}$ is higher in boys with hypospadias than those in prepubertal control subjects (29); and genetic variants of the LH and its receptor have been associated with disorders of the male reproductive tract $(30,31)$.

Not only endocrine regulation but also other ATF3'sdependent biological aspects may be disturbed by gene

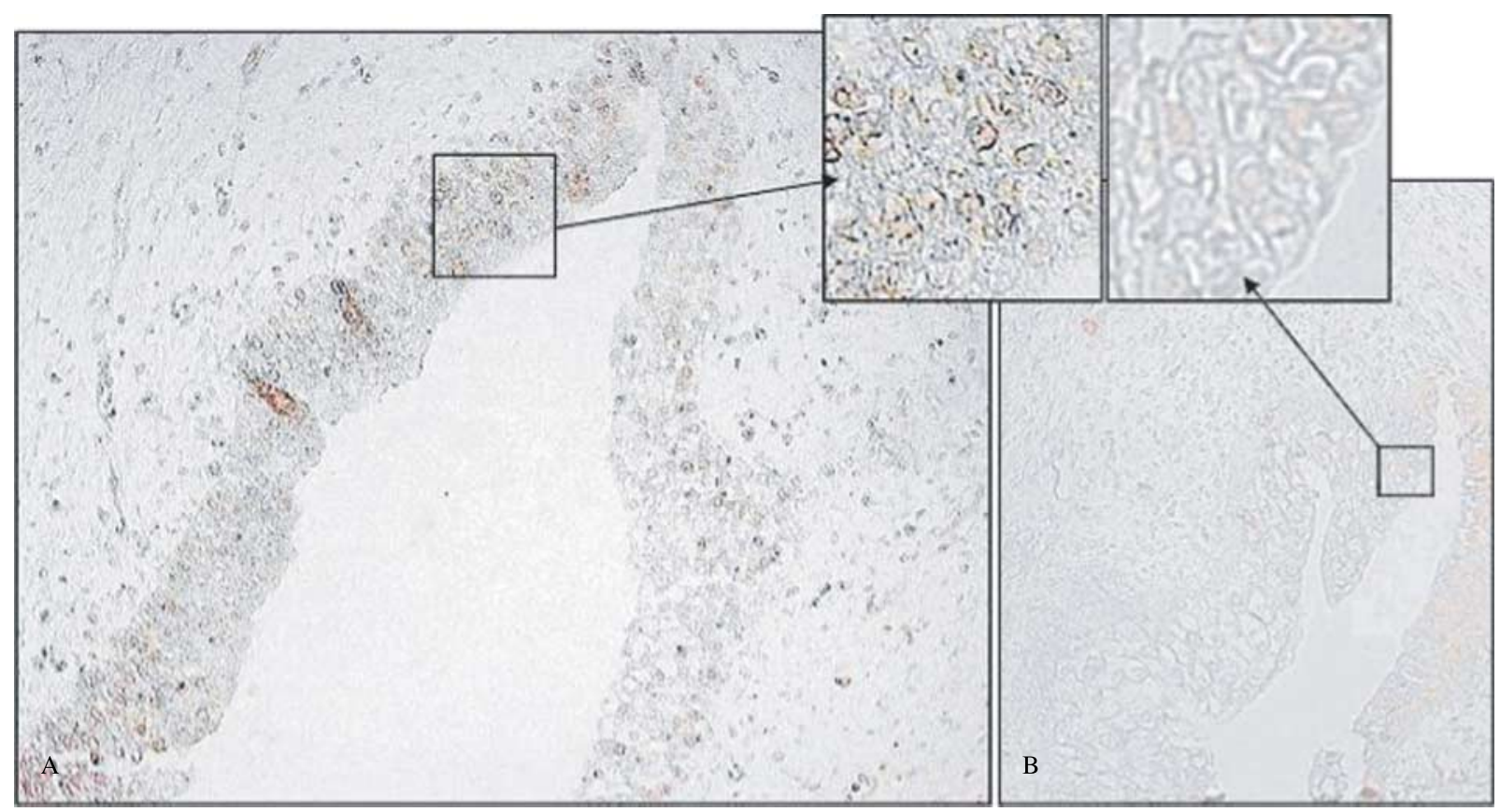

Figure 7 ATF3 expression by immunohistochemistry in posterior urethra from fetal gestation week 15. (A) ATF3 expression is seen in urethral wall, particularly in the epithelium, localizing in the nucleus preferentially located adjacent to the nuclear membrane; no expression was observed before the 15th week. Similar expression pattern is seen in female vagina from 9th to 15th gestational weeks. (B) A similar section of the developing male urethra at 11 weeks shows no ATF3 immunostaining (sections photographed at $400 \times$ magnification). 
variants in this gene. Indeed, ATF3 is normally expressed at a steady state in quiescent cells. It has traditionally been related to cell survival and/or apoptosis, stress, DNA damage, homeostasis, wound healing, cell adhesion, inflammation, nutrient limitation, and chemical exposure (32-36). Moreover, overexpression of ATF3 suppresses cell growth, by slowing the progression of cells from G1 to S phase, and it has been related to pro-apoptotic events and in the regulation of cell fate $(36,37)$. This is a relevant aspect due to the importance of cell proliferation and apoptosis regulation during development $(38,39)$.

Furthermore, ATF3 responds to signals in epithelial cells via the transforming growth factor- $\beta$ (TGF- $\beta$ ) pathway $(39,40)$. Interestingly, genes involved in the TGF- $\beta$ pathway that facilitates epithelial-mesenchymal interactions are up-regulated during several stages of urethral tube development in mice (12, 40-42). Moreover, ATF3 lies at the centre of interactions between the TGF- $\beta$ signaling pathway and steroid hormone receptors (12). Indeed, the normal male genital tubercle development depends on well-coordinated epithelial-mesenchymal interactions (2).

ATF3 seems to be involved in crucial aspects of urethral and genital developments. It is possible that variants in the ATF3 sequence and/or environmental mechanisms, such as endocrine disrupters $(9,10,12)$ and lower nutrient availability (35) (a link to low birth weight? (4)) lead to inappropriate regulation of ATF3, which might halter the seam fusion process of the developing urethra, by suppressing cell growth and/or promoting cell death, by disturbing hormonal signaling, or by impairing epithelial-mesenchymal differentiation, and result in a shorter urethra with a proximal opening.

In summary, the evaluation of ATF 3 as a candidate for hypospadias indicates that sequence variants of this gene, which is expressed in the human developing male urethra and may play a central role in urethral development, may be involved in the genetic risk for hypospadias in different ways: by the effect of three common low risk SNPs in the intron 1 of ATF3; or induced by less common variants, possibly with higher effect, such as the missense mutation c.536A $>\mathrm{G}$ in exon 3 and c. $817 \mathrm{C}>\mathrm{T}$ in the $3^{\prime}$-UTR.

\section{Acknowledgements}

This work was supported by grants from the Swedish Society for Medical Research, Swedish Research Council, HRH Crown Princess Lovisa Foundation, Karolinska Institutet, Erik Rönnberg donor (Riksbankens jubileumsfond), foundation Frimurare in Stockholm, the Swedish Society of Medicine, the Magn. Bergvalls and Fredrik and Ingrid Thurings foundations. The authors have no conflicts of interest.

\section{References}

1 Utsch B, Albers N \& Ludwig M. Genetic and molecular aspects of hypospadias. European Journal of Pediatric Surgery 200414 297-302.

2 Baskin LS, Erol A, Jegatheesan P, Li Y, Liu W \& Cunha GR. Urethral seam formation and hypospadias. Cell and Tissue Research 2001 305 379-387.

3 Fredell L, Iselius L, Collins A, Hansson E, Holmner S, Lundquist L, Lackgren G, Pedersen J, Stenberg A, Westbacke G \& Nordenskjold A. Complex segregation analysis of hypospadias. Human Genetics 2002111 231-234.

4 Fredell L, Kockum I, Hansson E, Holmner S, Lundquist L, Lackgren G, Pedersen J, Stenberg A, Westbacke G \& Nordenskjold A. Heredity of hypospadias and the significance of low birth weight. Journal of Urology 2002167 1423-1427.

5 Klonisch T, Fowler PA \& Hombach-Klonisch S. Molecular and genetic regulation of testis descent and external genitalia development. Developmental Biology 2004270 1-18.

6 Yucel S, Cavalcanti AG, De Souza A, Wang Z \& Baskin LS. The effect of oestrogen and testosterone on the urethral seam of the developing male mouse genital tubercle. BJU International 2003 92 1016-1021.

7 Crescioli C, Maggi M, Vannelli GB, Ferruzzi P, Granchi S, Mancina R, Muratori M, Forti G, Serio M \& Luconi M. Expression of functional estrogen receptors in human fetal male external genitalia. Journal of Clinical Endocrinology and Metabolism $2003 \mathbf{8 8}$ $1815-1824$.

8 Dietrich W, Haitel A, Huber JC \& Reiter WJ. Expression of estrogen receptors in human corpus cavernosum and male urethra. Journal of Histochemistry and Cytochemistry 200452 355-360.

9 Yucel S, Cavalcanti AG, De Souza A, Wang Z \& Baskin LS. The effect of oestrogen and testosterone on the urethral seam of the developing male mouse genital tubercle. BJU International 2003 92 1016-1021.

10 Kim KS, Torres CR, Jr, Yucel S, Raimondo K, Cunha GR \& Baskin LS. Induction of hypospadias in a murine model by maternal exposure to synthetic estrogens. Environmental Research 200494 267-265.

11 Beleza-Meireles A, Kockum I, Lundberg F, Soderhall C \& Nordenskjold A. Risk factors for hypospadias in the estrogen receptor 2 gene. Journal of Clinical Endocrinology and Metabolism $2007923712-3718$.

12 Willingham E \& Baskin LS. Candidate genes and their response to environmental agents in the etiology of hypospadias. Nature Clinical Practice Urology 20074 270-279.

13 Baskin LS, Colborn T \& Himes K. Hypospadias and endocrine disruption: is there a connection? Environmental Health Perspectives 2001109 1175-1183.

14 Giwercman A, Rylander L, Hagmar L \& Giwercman YL. Ethnic differences in occurrence of TDS- genetics and/or environment? International Journal of Andrology 200629 291-297.

15 Wang Z, Liu BC, Lin GT, Lin CS, Lue TF, Willingham E \& Baskin LS. Up-regulation of estrogen responsive genes in hypospadias: microarray analysis. Journal of Urology 2007177 1939-1946.

16 Liu B, Wang Z, Lin G, Agras K, Ebbers M, Willingham E \& Baskin LS. Activating transcription factor 3 is up-regulated in patients with hypospadias. Pediatric Research 200558 1280-1283.

17 Hai T, Wolfgang CD, Marsee DK, Allen AE \& Sivaprasad U. ATF3 and stress responses. Gene Expression 19997 321-335.

18 Liu B, Agras K, Willingham E, Vilela ML \& Baskin LS. Activating transcription factor 3 is estrogen-responsive in utero and up-regulated during sexual differentiation. Hormone Research $200665217-222$.

19 Mall FP. On measuring human embryos. Anatomical Record 19071129.

20 Moore KL. The Developing Human. 3 edn. Philadelphia: W B Saunders Co., 1982.

21 Patten BM. Human Embryology. 3 edn. New York: McGraw Hill Book Co., 1968. 
22 Povey S, Lovering R, Bruford E, Wright M, Lush M \& Wain H. The HUGO Gene Nomenclature Committee (HGNC). Human Genetics 2001109 678-680.

23 den Dunnen JT \& Antonarakis SE. Mutation nomenclature extensions and suggestions to describe complex mutations: a discussion. Human Mutation 200015 7-12.

24 Woolf B. On estimating the relation between blood group and disease. Annals of Human Genetics 195519 251-253.

25 Barrett JC, Fry B, Maller J \& Daly MJ. Haploview: analysis and visualization of $\mathrm{LD}$ and haplotype maps. Bioinformatics 200521 263-265.

26 Dudbridge F. Pedigree disequilibrium tests for multilocus haplotypes. Genetic Epidemiology 200325 115-121.

27 Pelzer AE, Bektic J, Haag P, Berger AP, Pycha A, Schafer G, Rogatsch H, Horninger W, Bartsch G \& Klocker H. The expression of transcription factor activating transcription factor 3 in the human prostate and its regulation by androgen in prostate cancer. Journal of Urology $2006 \mathbf{1 7 5} 1517-1522$.

28 Xie J, Bliss SP, Nett TM, Ebersole BJ, Sealfon SC \& Roberson MS Transcript profiling of immediate early genes reveals a unique role for activating transcription factor 3 in mediating activation of the glycoprotein hormone alpha-subunit promoter by gonadotropinreleasing hormone. Molecular Endocrinology $2005 \quad 19$ 2624-2638.

29 Nonomura K, Fujieda K, Sakakibara N, Terasawa K, Matsuno T, Matsuura N \& Koyanagi T. Pituitary and gonadal function in prepubertal boys with hypospadias. Journal of Urology 1984132 595-598.

30 Pals-Rylaarsdam R, Liu G, Brickman W, Duranteau L, Monroe J, El-Awady MK, Gad YZ \& Shenker A. A novel double mutation in the luteinizing hormone receptor in a kindred with familial leydig cell hypoplasia and male pseudohermaphroditism. Endocrine Research 200531 307-323.

31 Themmen AP \& Brunner HG. Luteinizing hormone receptor mutations and sex differentiation. European Journal of Endocrinology $1996 \mathbf{1 3 4} 533-540$.

32 Fan F, Jin S, Amundson SA, Tong T, Fan W, Zhao H, Zhu X, Mazzacurati L, Li X, Petrik KL, Fornace AJ, Jr, Rajasekaran B \& Zhan Q. ATF3 induction following DNA damage is regulated by distinct signaling pathways and over-expression of ATF3 protein suppresses cells growth. Oncogene 200221 7488-7496.

33 Wolfgang CD, Liang G, Okamoto Y, Allen AE \& Hai T. Transcriptional autorepression of the stress-inducible gene ATF3. Journal of Biological Chemistry 2000275 16865-16870.
34 Kawauchi J, Zhang C, Nobori K, Hashimoto Y, Adachi MT, Noda A, Sunamori M \& Kitajima S. Transcriptional repressor activating transcription factor 3 protects human umbilical vein endothelial cells from tumor necrosis factor-alpha-induced apoptosis through down-regulation of p53 transcription. Journal of Biological Chemistry 2002277 39025-39034.

35 Pan YX, Chen H, Thiaville MM \& Kilberg MS. Activation of the ATF3 gene through a co-ordinated amino acid-sensing response programme that controls transcriptional regulation of responsive genes following amino acid limitation. Biochemical Journal 2007 401 299-307.

36 Guerra S, Lopez-Fernandez LA, Garcia MA, Zaballos A \& Esteban M. Human gene profiling in response to the active protein kinase, interferon-induced serine/threonine protein kinase (PKR), in infected cells. Involvement of the transcription factor ATF-3 IN PKR-induced apoptosis. Journal of Biological Chemistry $2006 \mathbf{2 8 1}$ 18734-18745.

37 Fan F, Jin S, Amundson SA, Tong T, Fan W, Zhao H, Zhu X, Mazzacurati L, Li X, Petrik KL, Fornace AJ, Jr, Rajasekaran B \& Zhan Q. ATF3 induction following DNA damage is regulated by distinct signaling pathways and over-expression of ATF3 protein suppresses cells growth. Oncogene 200221 7488-7496.

38 Baskin LS, Liu W, Bastacky J \& Yucel S. Anatomical studies of the mouse genital tubercle. Advances in Experimental Medicine and Biology 2004545 103-121.

39 Morgan EA, Nguyen SB, Scott V \& Standler S. Loss of Bmp7 and Fgf8 signaling in HOXA13-mutant mice causes hypospadia. Development 2003130 3095-3109.

40 Valcourt U, Kowanetz M, Niimi H, Heldin CH \& Moustakas A. TGFbeta and the Smad signaling pathway support transcriptomic reprogramming during epithelial-mesenchymal cell transition. Molecular Biology of the Cell 200516 1987-2002.

41 Kang Y, Chen CR \& Massague J. A self-enabling TGF-beta response coupled to stress signaling: Smad engages stress response factor ATF3 for Id1 repression in epithelial cells. Molecular Cell 200311 915-926.

42 Massague J, Seoane J \& Wotton D. Smad transcription factors. Genes and Development $2005192783-2810$.

Received 17 December 2007

Accepted 1 February 2008 